\title{
Maurice Barrès et l'icône de l'art moderne
}

\author{
Boštjan Marko Turk
}

\author{
L'auteur dédie cet article à la mémoire d'Ante Glibota, vice-président \\ de L'Académie Européenne des Sciences, des Arts et des Lettres.
}

\section{Synopsis}

La peinture d'El Greco remplissait Barrès d'étonnement et d'admiration parce quelle répondait à la manière dont l'écrivain appréhendait le culte du fort et de l'exceptionnel. C'est la quête de l'absolu qui perfectionne les êtres dominés par l'unique tendance - vivre dans l'exaltation du moi jusqu'à ce qu'ils ne fusionnent avec l'absolu. Pour cette raison Barrès était capable de saisir le génie multifonctionnel du peintre grec et de transmettre son message à la postérité. Barrès approchait le peintre d'une perspective multilatérale : il s'apercevait des traits maniéristes. Ceux-ci sont devenus élément intégral de la peinture moderne, notamment chez Pablo Picasso. Les périodes bleue et rose n'auraient pas été possibles sans la rencontre d'El Greco.

Mots-clefs : El Greco, Maurice Barrès, Tolède, les saints, le culte du moi, les déracinés, le maniérisme, l'orthodoxie, les icônes, la théophanie, Pablo Picasso. 
Interest in his work returned, sporadically at first with nineteenth century Romanticism: and in the present century as a widespread, enthusiastic taste, stimulate with modern art with its great freedom and intensity. The writings of the Spanish scholar Cossio, ${ }^{1}$ the German critic Meier-Graefe ${ }^{2}$ and the French novelist and essayist Barrès were influential at El Greco's revival (Matthews, 1953, 14).

John F. Matthews, dans la prestigieuse édition «Library of Great Art », évoqua Maurice Barrès comme un des promoteurs de l'artiste dont l'influence aurait été prépondérante chez des artistes comme Picasso (et Pollock). ${ }^{3}$

Les sources de l'affinité qui mettraient le peintre et l'écrivain dans la même "communauté de l'être " (Jousset, 2009, 122) se dévoilent per se. L'explication s'offrirait par le biais de la prédilection que Maurice Barrès, lors de son adhérence au beylisme, vouait aux consciences fortes, parmi lesquelles étaient des saints, surtout dans leur rapport au Vivant éternel. En fait, on les admire puisqu' ils effectuaient la convenance nec plus ultra de la volonté à la loi morale.

Les héros de ce genre se font remarquer par l'énergie particulière qui exalte la tendance personnelle à s'affirmer indépendamment des autres. Ils se dépassent en repoussant leurs limites. Leur conscience tend vers un niveau supérieur de la réalité où ils s'approchent de l'inconcevable tout en demeurant dans la finitude du corps et de la pensée.

Barrès cultivait une préférence pour les caractères hors du commun. De là, sa prédilection pour Stendhal et l'expression qu'il a lui-même forgée, l'égotisme. ${ }^{4}$ L'égotisme fait la partie indélébile de la première étape de sa création où l'écrivain se voue à une méditation qui aboutit à la trilogie Le Culte $d u$ Moi. La triade se

1 Cossio, Manuel. El Greco. Madrid : Victoriano Suarez, 1908.

2 Meier-Graefe, Julius. Spanische Reise. Berlin : Fischer Verlag, 1910.

3 On donne la chronologie des rencontres entre Maurice Barrès et l'objet de son livre. À voir le texte cité d'après "L'Introduction » (Rambaud, 1994) à El Greco ou le Secret de Tolède (Barrès, 1994), on remarquera que Barrès commença tôt à s'intéresser au peintre et que ses écrits sur le sujet coïncident avec la monographie publiée par Manuel B. Cossio en 1908 (Cossio, 1908) à qui on accorde volontiers le primat de la remise à jour de l'œuvre recouverte de l'ombre. Ce n'est qu'après une lecture approfondie qu'on voit que les lumières que jette Maurice Barrès sur le phénomène plus qu'opaque pénètrent plus en profondeur que ne le font les textes de Cossio (Cossio, 1908) et de Meier-Graefe (Meier-Grefe, 1910). Ainsi : "La passion de Barrès pour Tolède date de son premier séjour en Espagne en 1892 (...) La première mention du Greco dans Mes cahiers date seulement du printemps 1899. Dès 1901, en revanche, il annonce une "étude sur Greco", alors que ce n'est qu'au cours d'un séjour à Tolède qu'il découvrira, en compagnie du peintre espagnol Beruete, les principaux tableaux de l'élève de Tintoret. D'avril à septembre 1909, il publie en tout cas une série d'articles qui compose déjà l'essentiel de Greco ou le Secret de Tolède. Dans Greco ou le Secret de Tolède qui suivra en 1912 et comprendra le seul texte de Barrès, on peut lire : "Je n'ai que trop attendu! " " (Rambaud, 1994, 511-512).

4 Le mot est composé du pronom personnel latin de la première personne du singulier, ego, et le suffixe indiquant la généralisation. 
compose des romans : Sous l'cil de Barbares, Un Homme libre et Le Jardin de Bérénice (Barrès, 1994).

Barrès cherchait en lui-même le principe de l'épanouissement et cultivait méthodiquement les sensations. Puis à un moment donné, il a renoncé à l'attitude individualiste : il s'est jeté dans les luttes « du moment » exaltant l'énergie nationale, la force de l'État et de l'Église.

Ceux que Barrès considérait comme les plus forts étaient les saints. Un saint qui n'est pas capable de résister à la nature qui demande sa disparition serait une antinomie, une contradiction. Il n'y a pas de saints qui soient morts dans le sens le plus commun du mot. Conformément à la tradition ceux qui revêtirent le caractère sacré avaient dû au préalable passer par un entraînement dur et tenir compte de la discipline, de l'hygiène spirituelle : ils se sont livrés à un apprentissage par l'habitude qui consistait en " cultivation » et en " transformation » du « moi », celui-ci est devenu, à force de discipline, une entité invincible, ouverte aux arcanes de l'éternel. Le « moi » n'est donc « égotique » que dans la mesure où il tend à échapper à la tiédeur - «Ainsi, parce que tu es tiède, et que tu n'es ni froid ni bouillant, je te vomirai de ma bouche " (Apocalypse 3 :16) -, au gris, au manque de fièvre.

Le « moi » aurait l'ambition de laisser derrière tout ce qui l'empêche d'embrasser l'enthousiasme de l'éternel. Ainsi :

Les ordres religieux ont créé une hygiène de l'âme qui se propose d'aimer parfaitement Dieu; une hygiène analogue nous avancera dans l'adoration du Moi. C'est ici, à Saint-Germain, un institut pour le développement et la possession de toutes nos facultés de sentir; c'est ici un laboratoire de l'enthousiasme. Et non moins énergiquement que firent les grands saints du christianisme proscrivons le péché qui est la tiédeur, le gris, le manque de fièrure, c'est-à-dire tout ce qui contrarie l'amour (Barrès, 1922, 39).

La peinture d'El Greco remplissait Barrès d'étonnement et d'admiration parce qu'elle répondait aux appréhensions par lesquelles l'écrivain jugeait le culte du fort et de l'exceptionnel. C'est la quête de l'absolu qui perfectionne les êtres dominés par l'unique tendance - vivre dans l'exaltation de leur moi et jusqu'à ce qu'ils ne fusionnent avec l'absolu. El Greco, notamment s'identifie aux figures qu'il peint : "Avec un magnifique sang-froid, il élimine tout ce qui n'est pas l'essentiel, et il s'élance violemment vers ce qui et pour lui l'absolu " (Barrès, 1994, 544). La façon dont il peignait fut à l'échelle de son intention : il tendait à adapter les personnages aux postures qui traduisaient leur passage de l'espace humain à la communion du Christ. Cela demanda une technique appropriée.

Maurice Barrès s'en est aperçu. A lire attentivement les passages où il décrit le répertoire dans lequel puisait le Grec, on voit qu'il est bien conscient des formules dont se servait le peintre afin de mettre en œuvre son ambition. 
Qui croirait que Domenico Greco esquissât ses ouvrages, les retouchât à maintes reprises, afin de séparer et désunir les teintes, pour donner ainsi à ses toiles leur aspect de cruelles ébauches, et pour simuler une plus grande liberté de fracture, une plus grande puissance. (...) El Greco se préoccupait d'éviter le rondouillard et cherchait l'expression crue. (Barrès, 1994, 526).

Le passage cité pourrait être corroboré par un autre visant la même idée : « Ses sculptures aux formes sveltes, parfois même quintessenciées et subtiles, témoignaient d'une passion concentrée " (Barrès, 1994, 522-523).

Maurice Barrès s'étonnait de la force par laquelle Le Grec disposait les formes humaines afin de trouver une expression qui aurait satisfait son désir de se libérer en écartant les limites de l'ordinaire. Le Crétois était en quête singulière d'une "plus grande puissance ", "d'une plus grande liberté de fracture ». ${ }^{5}$ L'écrivain s'est aperçu que le chemin qu'empruntait El Greco passait par la tendance d'amener ses objets au plus haut point de subtilité ; qu'il les raffinait avec un soin particulier; que ses couleurs étaient troubles et obscures, à vrai dire « crues »; que l'espace dans lequel il replaçait les narratifs de ses tableaux était désuni pour ne pas dire indéfini et que finalement ses figures subissaient la torsion du corps, réduites à "l'état du spasme qui nous surprend et nous anime " (Barrès, 1994, 544). Le jeu artistique dont prenait conscience Barrès est synonyme du style maniériste : c'est un outil quicombiné aux autres - définit l'identité du peintre.

En fait, à l'égard de l'œuvre picturale d'El Greco, on reconnaît les formes serpentines aux aspects légers et élancés, les flammes bondissantes qu'adoptent les martyrs et les saints lorsque, soutenus par le prodige surnaturel, ils rejoignent l'au-delà chrétien qui devient leur demeure ultime. Ils subissent l'altération de la figure humaine : l'architecture qui détermine le corps du vivant se perd.

Dans ces travaux des années 1580, le peintre rejette de plus en plus les lois de la perspective et des proportions énoncées par la Renaissance. Ses personnages perdent leur relief, s'allongent et s'amincissent. Pacheco nous apprend qu'El Greco ne préparait pas ses compositions avec des modèles vivants, comme Tintoret, mais avec des figures en argile (Michael Scholz-Halsen, 2004, 38).

Le tableau auquel Maurice Barrès vouait une particulière admiration est L'Enterrement du Comte Orgaz. L'engouement n'était pas sans raisons : "Greco, ajustant mieux son but, allait, au lendemain de cette demi-réussite, ${ }^{6}$ atteindre son point de perfection dans le fameux Enterrement du comte d'Orgaz. Voilà son chef d'œuvre populaire. C'est la gloire » (Barrès, 1994, 522). En fait, tout Greco où l'allongement du corps

5 Cf. supra.

6 La toile qu'il a effectuée précédemment, Le Martyre de saint Maurice, ne semblait pas pleinement contenter le commanditaire, Philippe II. 
est en fonction de la médiation qui relie l'immanent au transcendent, est là. En plus, afin de peindre le passage au Ciel, l'artiste devait se servir du chromatisme qui ne semble pas appartenir au coloris terrestre :

Au même moment l'âme de Seigneur d'Orgaz reçoit audience de la Cour céleste. Cette audience nous la voyons. Elle occupe le ciel du tableau. Le seigneur d'Orgaz s'y présente tout nu devant le Christ, la Vierge et le cercle des Bienheureux. La scène fait un contraste absolu avec la belle peinture réaliste du bas. Des tons livides et restreints jusqu'à l'indigence, des formes prodigieusement allongées, amincies et tourmentées, lui donnent un caractère spectral qui nous inquiète, nous scandalise et nous attire (Barrès, 1944, 518).

C'est l'élite de la race humaine, tel le seigneur Orgaz, qui passe immédiatement au royaume des cieux et participe à l'existence pérenne : Maurice Barrès ne s'est jamais intéressé qu'aux couches "les plus considérées » où résident les élus, les génies du corps ou maîtres sans limite ne faisant aucune concession aux facultés intellectuelles et spirituelles. Il faut prendre cette constatation à la lettre : qu'un individu se soit anobli ou enrichi ne comptait en rien aux yeux de Barrès. Il avait une ambition supérieure : "l'univers réaliste du bas » ne l'intéressa que dans la mesure où il pourrait permettre la traversée vers l'éternité.

C'est en plein accord avec le tempérament d'un autre auteur qui exprima le même état d'âme par les alexandrins suivants :

J'ai de l'ambition, mais plus noble et plus belle: Cette grandeur périt, j'en veux une immortelle, Un bonheur assuré, sans mesure et sans fin, au-dessus de l'envie, au-dessus du destin. Est-ce trop l'acheter que d'une triste vie qui tantôt, qui soudain me peut être ravie; Qui ne me fait jouir que d'un instant qui fuit, Et ne peut massurer de celui qui le suit? (Corneille, 1963, 306-307).

Les vers du dramaturge sont synonymes à "la conquête de Dieu » (Doubrovsky, 1963, 222) dont la manifestation sont les toiles que Barrès admirait lors de ses séjours tolédans. En fait, Polyeucte témoigne de l'identité qui caractérise la famille d'esprit particulière, celle qui vise les objectifs élevés dans l'emploi absolu du terme. Le dénominateur commun qui les réunit relève de « la passion chrétienne. C'est cette querelle immense entre les amours de la terre et les amours du Ciel, que Corneille a peinte " (Chateaubriand, 1901, 281). C'est notamment l'état intellectuel, violent et puissant avec lequel E1 Greco a formé l'ensemble de l'Enterrement du comte Orgaz.

La mission qui a été accordée au personnage du premier plan est l'une des plus prestigieuses possibles. Le comte, par rapport à ses dispositions à faire du bien, voit au moment de sa sépulture apparaître saint Étienne et saint Augustin. Les 
deux sont les figures de proue de « l'Église militante », le premier représentant la tragédie de la chair qu'expriment les martyrs lorsque, sachant qu'aucun compromis n'est possible avec Dieu, ils passent au sacrifice absolu de leur nature, c'est-à-dire, à la mort. Saint Étienne a notamment obéi à la voix que Polyeucte avait entendue par les paroles de Néarque. De son côté, l'évêque d'Hippone connote le magistère de l'autorité spirituelle s'imposant de façon absolue. Leur rôle médiateur contribue à former un défilé ininterrompu où disposé en file, le drame de l'invocation de "l'être infini du verbe parfait " (Bruaire, 1964, 163) S'accomplit dans l'éternité anagogique, rendue concevable par les formes de cette « ininscriptible » peinture.

Lunivers de Barrès se plie à la loi du plus fort, du Saint des saints, solus Sanctus, de l'origine et de l'ineffable finalité qui réside au cœur du présent :

Aux yeux d'un contemporain de sainte Thérèse, les amis d'Orgaz sont des âmes assujetties à des corps. Elles séchappent de leurs gaines, flottent dans l'air, montent vers la gloire. C'est le geste du Christ, si doux, si élégant, qui les attire. Elles vont à lui comme les cours accourent à un mot sublime de poésie. Ainsi le génie du Greco parvient à nous rendre sensible la métaphysique qui enchante ses modèles (Barrès, 1994, 545-546).

Inspiré par la loi du plus fort, l'œuvre d'El Greco s'ouvre à la synthèse du maniérisme et de l'art byzantin. El Greco ne professait pas un simplissime catholicisme « unilatéral ». C'était un espace trop exclusif pour devenir source de son inspiration. Il était a priori un déraciné, ${ }^{7}$ considérant le terme d'un certain point de vue du roman qui annonce les idées de la trilogie Le Culte du moi.

Un moi devient idole, dans le sens étymologique, de tout ce qui existe et doit s'affirmer contre ce qui n'est pas lui-même. Il doit se " généraliser ». En poursuivant le plus général, il progresse vers la « nation » qui est un terme équivalent de l'objectivité de la culture " générale » (c'est-à-dire nationale) dans laquelle il s'enracine de nouveau. Pourtant la «culture » la plus générale, c'est celle qui provient de la notion la plus générale, qui est l'être. Dans l'ensemble de ses rapports dans le domaine de l'humain elle s'appelle la religion. Parce que l'homme n'ayant part à l'être qu'imparfaitement, monte vers le «nom substantiel » ${ }^{8}$, vers Dieu qui est l'être subsistant, puisqu'étant par soi : «Sum qui sum» (Exodus 3,14). En face de l'être le plus général l'individu éprouve une présence plus qu'aidante étant donné que celle-ci lui permet de s'épanouir intégralement. Plus l'homme est seul et plus il se sent jeté dans le milieu métaphysique où il habite, plus fort devient le lien qui le relie à la source de son être. Les individus déracinés sont particulièrement invités à le faire en pleine liberté parce qu'il n'y a rien qui pourrait les retenir.

$7 \quad$ Cf. : Barrès, Maurice. Les Déracinés. Paris : Charpentier, 1897.

8 L'expression est de saint Augustin. 
"Si nous en croyons Meier-Graefe, El Greco aurait puisé sa force dans le fait qu'il a été dépourvu de sa patrie » (Michael Scholz-Hansel, 2014, 88). ${ }^{9}$ Être un « déraciné » dans le sens dans lequel Barrès comprenait le vocable, sous-entendait un point de vue élevé sur les paradigmes qui, à l'époque, déterminaient l'art de la peinture et les différentes formes de la confession à la fois. Le credo d'El Greco était orthodoxe ce qui ne l'empêchait pas de devenir zélote catholique, dont l'ardeur était d'avantage accrue par la Contre-Réforme. Il admirait les docteurs de l'Eglise, tel sainte Thérèse d'Avila.

Tout simplement, c'est un catholique espagnol; je veux dire qu'il réalise une certaine qualité de sublime, que peuvent produire toutes les nations catholiques, mais auquel l'espagnol attache son nom. Ses toiles complètent les traités de sainte Thérèse et de saint Jean de la Croix (Barrès, 1994, 548).

Les tendances artistiques et spirituelles coexistaient dans son travail, sans entrer en contradiction mutuelle. Ce qui pourrait être conçu comme une aporie se révèle être la source fructueuse d'inspiration. Il s'agit d'une synthèse : elle s'est - à l'insu de l'auteur - portée garant de l'actualité de sa création, nonobstant les quatre siècles pendant lesquelles son art fut oblitéré.

El Greco ne fut ressuscité qu'à l'aube des temps modernes, ce qui a permis aux éléments maniéristes de sa peinture de renouer avec les tendances les plus audacieuses de l'avant-garde. Il y a deux périodes, bleue et rose dans l'œuvre du peintre majeur en ce qui concerne l'identité de l'art visuel au $20^{\mathrm{e}}$ siècle, inconcevable en dehors du contexte du Greco, tellement évidentes sont les analogies entre l'un et l'autre. Picasso et El Greco furent, les deux, étrangers. Ils ont été déracinés des cultures originaires de l'espace méditerranéen. L'état français a rejeté, après la guerre civile espagnole, la demande de passeport que Picasso lui avait adressée. Mal reçu à la Capitale, il allait s'installer sur la Côte d'Azur qui lui rappelait les paysages de son enfance passée en Espagne. Ce qui plus est :

Pablo Picasso a vécu lui-même aussi bien à Madrid qu'à Barcelone ce nouvel engouement pour El Greco. Et il se réfère sans cesse à ce dernier dans sa première phase artistique importante, la "période bleue " ce qui ne saurait nous surprendre. L'Enterrement de Casagemas ne serait pas pensable sans L'Enterrement du comte d'Orgaz. Le titre d'un de ses dessins de l'époque, Yo El Greco (Moi El Greco) nous dit à quel point il s'identifie lui-même au peintre grec (Michael Scholz-Hansel, 2014, 88).

9 Maurice Barrès, appuyé sur un talent imparable a fourni une explication de l'itinéraire d'El Greco d'une façon encore plus complète : "Homme étrange, qui double d'un personnage énigmatique le mystère de son art. Une vie et des ceuvres submergées par les ténèbres, tel est le sort du Greco " (Barrès, 1994, 523). 
Si extraordinaires que les parallélismes entre Picasso et le Grec paraissent, ils ne sont pas invraisemblables. Ayant fait connaissance de l'univers pictural de son prédécesseur, Pablo Picasso aurait sans doute remarqué les possibilités expressives frappantes des figures allongées non naturalistes. Il aurait vu comment El Greco a adapté l'échelle et la perspective à ses propres fins. Il aurait ressenti l'intensité et le cadrage claustrophobe de l'image. On pourrait aussi recourir à une autre explication du phénomène :

One of the early examples from Picasso's Blue Period, Burial of Casegamas mirrors the split composition of The Burial of Count Orgaz, as well as its palette. Paintings Picasso produced in this period all display expressive mannerisms, distortion of the body, and swirling dynamic compositions " (Demiray, 2012,10).

Dans la période rose il y a deux toiles qui ne seraient pas nées si la peinture tolédane n'avait pas existé, au préalable. Ce sont Les Demoiselles d'Avignon et L'Ouverture du cinquième Sceau de l'Apocalypse. Les deux sont précurseurs de la modernité : les analogies avec la peinture d'El Greco sont de nouveau surprenantes. Picasso, notamment aurait également vu comment les figures nues presque monochromes en arrière-plan, qui s'efforcent également de prendre leurs robes, dans la scène de L'Apocalypse, complétaient Saint Jean et aidaient à répandre l'énergie du moment sur la toile. Il est possible aussi que les robes de la Vision de Saint Jean et le jeu de lumière sur elles trouvent leur écho chez Picasso dans les rideaux contre lesquels les prostituées des Demoiselles d'Avignon posent.

Le réseau des ressemblances ne semble, à vrai dire, avoir de limites puisque le style d'El Greco est particulièrement ouvert et accueillant. Il se rend distinct par les images troubles et obscures qui échappent à la réalité. Il se caractérise davantage par un espace où la complétude des éléments constitutifs est suspendue, dissoute. C'est cela qui a permis à Picasso d'en faire une certaine imitation sans en fait être un imitateur. Le style d'El Greco a constitué un courant - invisible pour une certaine période - qui impliquait plus tard les solutions apportées par le modernisme et l'avant-garde du $20^{\mathrm{e}}$ siècle. Le maniérisme surtout ne se voulait pas être conforme à la réalité. Il était donc loin du pragmatisme réaliste et de la réalité ordinaire. Il était stendhalien, dans le sens particulier du mot.

C'est son style (et ses effets) qui a permis à Greco d'être idole en (de) soi : «On sait qu'El Greco se faisait de son art une haute idée » (Barrès, 1994, 526). Et :

Defying all the accepted beliefs and painting techniques with his visual language and arrogant personality, El Greco was completely unlike any other painter of his time (Demiray, 2012, 6). 
On retiendra surtout le jugement présomptueux par lequel E1 Greco expliquait le «talent apparent » de Michel-Ange. Il est vraiment surprenant de lire le passage suivant :

Un peintre et critique sévillan, nommé Pacheco demanda encore au Greco ce qui doit l'emporter du dessin ou de la couleur. Le vieux maître répondit que c'était la couleur, puis il déclara que "Michel-Ange était un bon homme, mais quill ne savait pas peindre. Ce quill faut entendre, je crois : "Michel-Ange est un véritable homme (au sens où Napoléon dit à Goethe: Vous êtes un homme, monsieur de Goethe), mais il ne fait pas proprement de la peinture, il dessine des groupes statuaires (Barrès,1994, 526).

Une telle audace n'a pas réussi à mettre en question l'œuvre du Florentin ; pourtant, elle a retenti à travers les siècles.

E1 Greco joignait les expériences qu'il recevait aux écoles italiennes de l'époque aux leçons qu'il s'était vu donner lors de son apprentissage en Crète. Celui-ci avait consisté en peinture des icônes dans la tradition byzantine orthodoxe. Il est à rappeler que dans la tradition du christianisme oriental les icônes sont des objets théophanes. ${ }^{10}$ Cela signifie que l'icône est transparente dans le sens de devenir reflet de la toute-puissance divine. Les icônes sont sacrées : elles transmettent une réalité théologique et expriment une pensée exégétique. Elles s'identifient alors aux saints qu'elles dépeignent. Toute icône devient nécessairement un objet de la transfiguration: par le biais de celle-ci elle est autre chose que ce qu'on voit puisqu'elle se réfere à l'invisible et à l'ineffable (à l'indicible), c'est-à-dire à l'actualité mystique des saints et de Dieu.

Une icône présuppose bien plus qu'un simple art pictural réalisé sur les panneaux de bois. Sa fonction est générale en ce qui concerne l'orthodoxie : elle est destinée à la dévotion dans les cercles fermés du clergé orthodoxe ou à l'exposition sur des cloisons ornées qui séparent la nef du sanctuaire. L'œuvre d'E1 Greco témoigne de la theophanie: ses figures disparaissent dans les cieux lorsque leurs corps prennent les formes serpentines, pareilles aux flammes. Il se peut que le peintre grec soit allé encore un pas plus loin, vers le maniérisme comnène, c'est-àdire, vers une variante de ce style sophistiqué qui est né dans l'empire byzantin au 12e siècle et dont l'archétype serait La Vierge de Vladimir. In ultima analysi : le style comnène présente un aspect du maniérisme où tout n'est que le reflet approfondi d'une spiritualité austère et intégriste.

Quoi qu'il en soit au début du 17e siècle la peinture d'El Greco semblait être destinée à l'oubli. Après la disparition physique de Domenikos Theotokopoulos ses œuvres sont passées à la trappe. Ce n'est qu'au début du $20^{\mathrm{e}}$ siècle que des artistes et des critiques commencèrent à s'intéresser à son art très personnel.

10 Le mot vient du grec : il est composé de theos et de phanien qui signifient « apparition de Dieu ». 
Ce sont les cartographes, les artistes, les ecclésiastiques, les humanistes, etc. qui avaient soutenu le peintre, et non les princes et leur cour, et ce furent les écrivains, les critiques d'art et une avant-garde artistique déjà internationale vers 1900 qui initièrent sa découverte - et non les historiens d'art (Michael Scholz-Hansel, 2014, 87).

Celui pourtant, dont l'influence quant à la propagation du Grec fut décisive, était Maurice Barrès. On doit à ces textes le respect de la lecture intégrale. Greco ou le secret de Tolède est - dans ce sens - un texte révélateur et moderne. Il est multiculturel avant la lettre: son auteur parle du superbe dialogue entre la culture chrétienne et arabe. C'est une leçon pluriethnique qui au temps de Barrès ne se faisait sentir que rarement. Ainsi :

Au milieu d'un pays immobile, elle forme aujourd'hui encore une énorme grappe, une ascension composite d'églises, de couvents, de maisons gothiques, de couloirs arabes haussés et rétrécis. Et ses pierres continuent de dire les mêmes choses qu'avait entendues El Greco et qu'il fortifie du discours abondant de ses tableaux dans les chapelles délabrées. Les raisons de Tolède! C'est un superbe dialogue entre la culture chrétienne et l'arabe qui s'assaillent et puis se confondent (Maurice Barrès, "Greco ou le secret de Tolède », in Romans et Voyages, Robert Laffont, Paris, 1994, p. 529).

Il est à rappeler que la rencontre entre Le Greco et Picasso était elle-aussi une rencontre multiculturelle, dans le sens complet du mot.

Les textes de Maurice Barrès sont pénétrés par l'esprit de la modernité puisqu'ils tiennent (Greco ou le secret de Tolède) compte des postulats qui régissent la coexistence multiethnique dans le monde globalisé d'aujourd'hui. Pourtant, l'écrivain, semble-t-il, a fait un effort plus que visible afin de délivrer un message plus général. La propension naturelle qu'il vouait à $\mathrm{El} \mathrm{Greco} \mathrm{était} \mathrm{parallèle} \mathrm{à} \mathrm{un}$ des instincts primordiaux, à la révolte contre la mort. El Greco a peint - sauf à quelques exceptions près - les êtres qui ont vaincu la mort ou s'apprêtent à le faire.

Xavier Bichat, un des pères de la médicine moderne, a défini l'essentiel de l'existence par la formule suivante : "La vie, c'est l'ensemble des fonctions qui s'opposent à la mort ». ${ }^{11}$ Maurice Barrès, le peintre qui l'a inspiré et l'art en général répondent au sentiment de l'angoisse qui est depuis l'éveil de la conscience la grande hantise de l'humanité. Nous sommes nés et nous disparaîtrons. C'est un fait accompli. Pourtant, dans le temps qui est à notre disposition nous profitons de nos avantages. Nous admirons l'art et les artistes. Nous sommes sensibles à leurs messages. C'est notre pérennité.

11 Cf. : https://dicocitations.lemonde.fr/citations/citation-2341.php, consulté le 20 septembre 2020. 


\section{BIBLIOGRAPHIE}

Barrès, Maurice. Les Déracinés. Paris : Charpentier, 1897.

Barrès, Maurice. Romans et Voyages. Paris : Robert Laffont, 1994.

Barrès, Maurice. Un Homme libre. Paris : Plon, 1922.

Bruaire, Claude. L'Affirmation de Dieu. Essai sur la logique de l'existence. Paris: Seuil, 1964.

Chateaubriand, François René de. Génie du Christianisme. Paris : Calmann-Lévy, 1901.

Corneille, Pierre. Euvres complètes. Paris : Seuil, 1963.

Cossio, Manuel. El Greco. Madrid : Victoriano Suarez, 1908.

Demiray, Gülfem. Picasso's Spanish Heritage: How El Greco is seen in cubism and in Picasso's works. Research paper, professor Jessica Winston, 2012.

Doubrovsky, Serge. Corneille et la dialectique du héros. Paris : Gallimard, 1963.

Jousset, David. Le Vocabulaire théologique en philosophie. Paris : Ellipses, 2009.

Matthews, John, F. El Greco. New York : Harry N. Abrams, 1953.

Meier-Graefe, Julius. Spanische Reise. Berlin : Fischer Verlag, 1910.

Michael Scholz-Hansel. El Greco. Köln : Taschen, 2004.

Scholz-Hansel, Michael. El Greco, le prophète des modernes. Köln : Taschen, 2014.

\section{Sources internet}

https://dicocitations.lemonde.fr/citations/citation-2341.php, consulté le 20 septembre 2020.

Boštjan Marko Turk, Université de Ljubljana Bostjan-marko.turk@guest.arnes.si

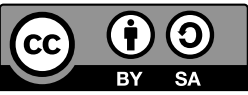

\section{Maurice Barrès in ikona sodobne umetnosti}

Maurice Barrès je bil med prvimi, ki je Evropi in svetu posredoval spoznanje o razsežnosti dela manierističnega slikarja El Greca. Dejansko je bil ta štiri stoletja v pozabi. Barrèsa je pri Grku pritegoval kult jaza in kult moči, kot se je v francoski književnosti oblikoval od Stendhala naprej. Francoski avtor je v svoji viziji Grecove umetnosti kult jaza spajal s kultom svetnikov, zmagovalcev nad smrtjo in absolutnimi favoriti življenja 
kot transcendenčne danosti. Zato je bil tudi bistveno motiviran sporočilo o umetniškem formatu slikarja posredovati dalje, vse do avantgarde, kjer je njegova slikarska vizija prišla polno do izraza. Modre in vijolične periode pri Pablu Picassu si namreč ne morem misliti brez El Greca.

Ključne besede: E1 Greco, Maurice Barrès, Toledo, svetniki, kult jaza, "ptički brez gnezda«, manierizem, pravoslavje, ikone, teofanija, Pablo Picasso.

\section{Maurice Barrès and the Icon of Modern Art}

El Greco's painting filled Barrès with astonishment and admiration because it responded to the writer's apprehensions regarding the cult of the strong and the exceptional. It is the quest for the absolute that perfects beings dominated by the only one tendency - to live in exaltation of their selves until they merge with the absolute. For this reason Maurice Barrès was able to grasp the multifunctional genius of the Greek painter and to transmit his message to posterity. Barrès approached the painter from a multilateral perspective: he saw the mannerist traits. These have become an element of modern painting, especially in Pablo Picasso. The blue and pink period would not have been possible without the encounter of El Greco.

Key words: E1 Greco, Maurice Barrès, Toledo, saints, worship of the self, mannerism, uprootedness, orthodoxy, icons, theophany, Pablo Picasso, 